ISSN: 2277-3754

ISO 9001:2008 Certified

International Journal of Engineering and Innovative Technology (IJEIT)

Volume 10, Issue 4, October 2020

\title{
Effect of the gap ratio on the flow patterns of the vortex flow forming behind the two square cylinders in tandem arrangement
}

\author{
Ramchandar Verma, Dheeraj Shriwas \\ Department of Mechanical Engineering, Patel College of Science and Technology, Indore
}

Special computer codes were created by Wahed et al [2] to

Abstract-This article discusses the effect of the gap ratio on the flux patterns of the vortex flow generated in the tandem arrangement of the two square cylinders in numerical simulation based on the square cylinder flow. This numerical simulation takes place fluently on ansys 14.5. This analysis is performed at number 150 of Reynolds. The study containing the simulation of the vortex drag phenomenon, force coefficient (drag and lift), the straw number with different lengths, pressure contours, speed contours, etc. CFD-simulation collects numerical results and contrasts them with previous experiments with the same number of Reynolds.It has found that vortex shedding for the spacing ratio of 4 is much similar to the shedding's occurred in the spacing ratio of 5. The nature of the shedding's in the spacing ratio of 1 and 2 have comparatively smaller vortex as compared to vortex formed in spacing ratio of 4 and 5 . The results also shows that the formation of the shadings are widely depends on the spacing ratio at fixed Reynolds number. Least shedding occurred at $S / D=3$.

Key Words - Vortex flow, Vortex Shedding, Force Coefficient, Strouhal Number, Spacing Ratio, Pressure Contours, Velocity Contours.

\section{INTRODUCTION}

Over the past few decades, the study on flow around bluff bodies has been the subject of mater for the intense research in the field of the vibrations and the components having the induced vibrations. In past few decades the area of the computational study has become faster and faster due to the involvement of computers- The computational capabilities of the computers make it easy to the researchers about the study around the bluff bodies. The aim of his study was to study the effect of induced vibrations on the cylinders used in the reactors and the reactor peripheral used in the reactors so that the vibrations can be reduce on the cylinders for the safer design of the reactors. Basically this study focuses on the reactors components that are in the vibrating condition in the reactor. These components are most important parts in the reactors which allow to withstand such high pressures. The flow induced vibrations are the most important on which each part of the reactor dependency is maintained. He found that the weakest part is the components having the two phase flow induced vibrations. The continuous exciting frequency of component produces the vortex shedding which makes it the most critical part of the reactors. The cross flow cylindrical arrays have been studied in this paper and analytical modelling has been done in this paper and concerned about the resonating buffeting of the component having the flow induced vibrations.

Manuscript received: 30 September 2020

Manuscript received in revised form: 28 October 2020

Manuscript accepted: 12 November 2020

Manuscript Available online: 15 November 2020 formulate the analytical model to calculate the nature of flow patterns formed in the turbulent flow regions. They utilize the Navier - stokes equation to understand the flow patterns obtained in the turbulent flow. These codes were used in the vortex flow meters to analyses the flow. In this study a Baldwin-Lomax turbulent model was used by them to understand the nature of the flows found in the vortex region. The aim of the study was to optimize the turbulent flows found in the Vortex shedding performance The study was then performed numerically and Experimental data was compared to the findings. Their comparison shows that the bluffing body type was the principal parameter to understand the flow characteristics produced by the flow. The comparison between Strouhal and test data helps them to infer the findings.

Flow vortex shedding formed were very distinct in the nature and it's all due to the shape of the bluff body encountering the flow. There were two types of the shedding's were formed called primary and the secondary shedding. The primary shedding was larger in nature as compare to the secondary shedding were formed in the near wake region of the body.

Norberg [3] presented an experimental study on the two rectangular cylinders. The experimental study on the two rectangular cylinders which were non-vibrating in nature was used. The two rectangular cylinders were fixed (non-vibrating in nature to investigate experimentally. The different angles or direction flows were utilized to investigate experimentally. The range of the variation in the angle of attack (i.e. the angle of encountering the flow) was in the range of zero to 90 degrees. The main aim of the study was to investigate the nature of tile flow patterns around the rectangular cylinders and the pressure forces round the cylinders. The forces that are trying to lift the body (i.e. the forces of lift) were the main target of the study. The side ratios of the study were as follows: $1,1.62,2.5$, and 3.The shortest side of the rectangle is $20 \mathrm{~mm}$. The hot wire measurement techniques were used to determine the flow patterns and pressure distribution around the rectangular cylinders. The static pressure distribution at the mid span was measured by the hot wire technique.

The frequency of the flow of wake and the Strouhal number were measured by the hot wire technique in the near wake regions. The smaller cylinders with 12 side ratios used in this study. The range of the side ratios lie in the range 1 to 5.The Reynolds number used in the study of the rectangular cylinders were taken in the range of $\mathrm{Re}=400$ to $3 \times 10^{4}$. The 


\section{ISO 9001:2008 Certified \\ International Journal of Engineering and Innovative Technology (IJEIT) \\ Volume 10, Issue 4, October 2020}

free stream turbulent intensity was less than $0.06 \%$.The blockage was less than $5 \%$ and the aspect ratio was L/A was greater than 50. For small the multiple wake frequencies were found and for the intermediate angle of attack the Strouhal number and the drag coefficient were approximately constant.

$\mathrm{Yu}$ and Kareem [4] they investigated on the rectangular cross-section rigid two dimensional prisms numerically. They used a large eddy simulation technique to investigate on the rectangular cross sectioned prism. The Reynolds number utilized in the study was taken as $10^{5}$. Thay used a finite difference method to analyses the grid solutions on the prism. The outer boundary conditions were satisfied by the convection conditions. The large eddy simulation (LES) technique unable them to predict the flow at very small sized structures also. This technique of large eddy simulation allow them to measure the velocity of the flow at any period of time and also the pressure differences that create during the flow over the structure taken to study. They also presented the pressure fluctuations and the aerodynamic forces such as the lift force and the drag forces on the surface of the prism. The Eigen functions of the pressure distribution around the surface of the prism also presented in this study. The streak lines are also employed to study around the surface of the prism. The streak lines patterns helps in the study of the shedding formed behind the rectangular sectioned prism which enables them to predict about the lift forces around the bluff body. They also investigated about the vorticity formed in the structures of the flow around the prism body. The obtained results were matched with the tunnel results which show good correlations. The streak line shows a good structure of the vortices formed having a reattachment zone around the prism. The pressure distribution on the faces of the rectangular prism having the side in flow of the fluid shows a constant distribution around it. But for the different separated regions the pressure distribution varies. The results simulated were the approximately same as the experimental results.

Saha et al [5] presented study around the square cylinder having the position placed centrally in the parallel plate. This study was done using the numerical simulations where the equations are solved by the software's used. The Reynolds number used for the study was taken as the 21400 over which whole study was concentrated. They make essential efforts to capture the turbulence quantities such as the vortex formation, stream lines etc. They used the three turbulence model to investigate numerically, The first model is the standard K- $\S$ model, second is Kato-Launder k- $\S$ model and the third one is RNG K- $\S$ model to perform the study. The results found by the numerical simulations were compared with the experimental data's at the same Reynolds number, The results were obtained for all the three models used and the Strouhal number is found for each. They found that the vortex shedding at The Strouhal number is the same for the entire model types used in the study. In all the three models they found that the Kato-Launder $\mathrm{k}-\S$ model is the best among all the models used. The time average velocities were found and compared with the experimental data's. A clear vortex shedding phenomenon was observed in the study. at the low Reynolds number the reattachment zone were observed. The high Reynolds numbers the reattachment Ones were not seen clearly.

Taylor and Vezza [6] investigated over the rectangular cross sectioned cylinders at the unsteady conditions. For the study around the rectangular cross sectioned cylinders the discrete vortex method was used. The study was done numerically over the rectangular cross-section cylinders in the unsteady wake. The basic of the numerical investigation was to take the discretization of the vortex field and not the velocity field. The results were plotted for the mean drag force, lift forces, Strouhal number and the various quantitative fields such as the vortcity fields, velocity fields etc. A discrete vortex method was employed to investigate numerically. The results were quite similar to the experimental data's.

Saha et al [7] presented a study Over the flow past the dimensional square cylinders in the static condition. They used a Reynolds number of 100 to perform the study over the two dimensional square cylinders in the static condition. They also used a finite difference scheme to study over two dimensional square cylinders in the static condition. A strong periodic moment was seen in the two dimensional square cylinders in the static condition placed in the channel centrally. The instantaneous flow field was visualized to understand the flow patterns over the two dimensional square cylinders in the static condition. The results simulated were the approximately same as the experimental results obtained at the high Reynolds number. The time average velocities were found and compared with the experimental data's. A clear vortex shedding phenomenon was observed in the study. at the high Reynolds number and the reattachment zone was observed. The instantaneous flow field was inherent in nature. The attenuation of peak vorticity was observed in the two dimensional square cylinders in the static condition at high Reynolds numbers. A very important result found was that the formation of the unsteady periodic oscillation in the wake which was similar to the wake structure formed in the turbulent flow over two dimensional square cylinders in the static condition. The decay rate of the circulation was seen in the downstream zone.

Liu and Chen [8] presented an experimental investigation on the two tandem square cylinders. The characteristic of flow around two tandem square cylinders were seen in the study with the experimental set of the cylinders. The range of the Reynolds number taken for the two tandem square cylinders in experiment varies from $2.0 \times 10^{3}$ to $1.6 \times 10^{4}$. The spacing ratio between the two stream wise square cylinders were in the range of the 1.5 to 9 .The hysteresis associated with the flow patterns on two tandem square cylinders were also studied. It was found that the hysteresis shifts towards the smaller values of the spacing ratio at higher Reynolds number. The time average history were found and compared with the experimental data's on experimental investigation on the two stream wise square cylinders. The results were plotted for the mean drag force, lift forces, Strouhal number 
ISO 9001:2008 Certified

International Journal of Engineering and Innovative Technology (IJEIT)

Volume 10, Issue 4, October 2020

and the. Various quantitative fields such as the velocity fields, velocity fields etc. The results were quite similar to the experimental data's and examined for the effects of hysteresis.

Kumar and Gowda [9] presented an experimental study on the flow induce vibrations over a square cylinder with or without the interferences. The interference effects were seen on the rigid square which was exposed to the flow. The number of arrangements use was tandem, side-side and staggered to study the interferences. The number of experiments was carrying out for the variation in the geometrical parameter of the test cylinder on the flow induce vibrations over a square cylinder. The variation in the relative dimension and spacing give rise to the larger amplitude vibrations on the experiment of flow induced vibrations over a square cylinder. The maximum amplitude found in the arrangement of the tandem at the spacing ratio of 1.25 and side ratio of 0.5 Flow visualization studies were conducted on the flow induced vibrations over a square cylinder at various arrangements. The results were plotted in terms of multiple amplitude of vibrations in study of flow over a square cylinder at various arrangements.

Dhiman et al [10] presented study over the effects of the Reynolds and the Prandlt number on the heat transfer across the square cylinder in the steady flow condition. The range of the Reynolds number was taken from 1 to 45 . The range of the Prandlt number was taken from 0.7 to 4000 in the cross flow. The cylinder temperature was kept constant and the boundary conditions were applied to the cylinder. The graph between the local Nusselt number and the isotherms were plotted at each part of the obstacle across the square cylinder. The local Nusselt number was found to be increasing with increase in the Reynolds number. The Nusselt number for the surface of the upstream cylinder has the highest value in the two dimensional steady flow regions across the square cylinder.

Kumar et al [11] presented numerical simulation over a square cylinder at low Reynolds number in a row of nine square cylinders arranged in a row. The value of the Reynolds number taken for the study was equal to 80 . The method used to perform the study was lattice-Boltzmann method. The effect of the cylinder spacing was studied over the arrangement ratio of the 0.3 to 12 of the diameter of the square cylinder. The results were plotted for the mean drag force, lift forces, Strouhal number and the various spacing ratio. The decrease in the spacing causes to increase in the mean drag coefficient and the strouhal number. The strength of the latter frequency depends upon the cylinder spacing. For larger range of the frequency the Strouhal number based on the gap velocity becomes almost constant. The flow quantities such as the flow regimes are plotted for primary and the secondary vortex formation. There was no significance in the interaction between the wakes observed for the spacing range of greater than six times the diameter of the cylinder.

\section{COMPUTATIONAL FLUID DYNAMICS} ANALYSIS

The popular method can be used to solve simple fluid flow equations. This includes the easy-to-resolve algebraic equations. However the essence of the fluid flow also helps you to understand the fluid flow's behaviour. Often if fluid is easy to manually flow, the simple differential equation is involved in nature. But it is simple to solve with the participation of the partial difference equation. Many researchers seek to find the system behaviour, in order to understand the relationship between the system variables, most of whom are experiments by engineers. Certain engineers attempt to numerically predict the device. Computational Fluid Dynamics (CFD) is such a tool to solve numerical equations.

The three basic steps of the research in the study of flow behind the bluff bodies are:

1. Next, define the problem area for which the analysis must be conducted. The pre-processing portion of these steps is named. This is used to describe the physics of the problem.

2. The solver is determined in this step through the use of software like fluid analysis - i.e. boundary conditions for fluid properties, flow models etc.

3. The data is used to trace contours, streamlines, vectors, etc, after all the results are achieved.

\section{METHODOLOGY}

Accessible literatures have been investigated and selected for unique Reynolds, i.e. $\operatorname{Re}=150$ for the analysis. The analysis geometry was built in GEOMETRY DOMAIN ANSYS 14.5, and MESH Generated was fluently launched in. For the analysis, different input parameters are defined. The findings are plotted with the Reynolds number at different distances.

\section{RESULTS AND DISCUSSION}

For the Reynolds re $=150$ range of the two square tubes, an analysis is done using the Ansys fluent version 14.5 with a separation range of 1 to $\mathrm{D} / \mathrm{S} / \mathrm{D}$ to 5 . The results are graphically illustrated and the values are reported and displayed in table. A time-step size $=03.01569$ is taken to capture the flow pattern clearly. Simulations are run for "700" iteration so that the time becomes 10 seconds. The flow time can be estimated as

Flow time $=$ Time-step size $\mathrm{x}$ Number of iterations

Flow time $=0.01569 \times 700$

Flow time $=10$

The Flow Visualization of the vortex shedding is done through contours of stream function, stream wise velocity, vorticity contours and static pressures. Instant flow patterns and other quantitative details are gathered to better understand the dynamic flow characteristics of the cylinders. Estimation of Drag Forces on two Cylinders at Various Spacing Ratios.

Table 1. Estimation of Drag Forces on two Cylinders at Various Spacing Ratios.

\begin{tabular}{|c|l|l|l|l|}
\hline S/D ratio & $\mathbf{C d}_{\mathbf{1}}$ & $\mathbf{C d}_{\mathbf{2}}$ & $\mathbf{C l}_{\mathbf{1}}$ & $\mathbf{C l}_{\mathbf{2}}$ \\
\hline 1 & 1.44 & -0.23 & \pm 0.01 & \pm 0.035 \\
\hline
\end{tabular}


ISSN: 2277-3754

ISO 9001:2008 Certified

International Journal of Engineering and Innovative Technology (IJEIT)

Volume 10, Issue 4, October 2020

\begin{tabular}{|l|l|l|l|l|}
\hline 2 & 1.42 & -0.25 & \pm 0.002 & \pm 0.007 \\
\hline 3 & 1.38 & -0.19 & \pm 0.001 & -0.002 \\
\hline 4 & 1.47 & 1.37 & \pm 0.050 & \pm 2.0 \\
\hline 5 & 1.50 & 1.63 & \pm 0.02 & \pm 2.45 \\
\hline
\end{tabular}

Comparison b/w $\mathrm{Cd}_{1} \& \mathrm{Cd}_{2}$ w.r.t. S/D

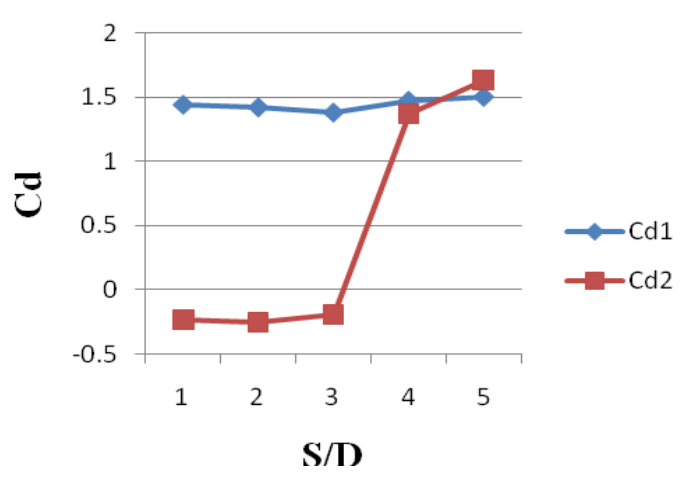

Fig 1 Comparison b/w $\mathrm{Cd}_{\mathbf{1}} \& \mathrm{Cd}_{\mathbf{2}}$ w.r.t. S/D

It is clear from the Table-.1 that drag coefficient on cylinder 1 is slightly decreasing in the range $1 \leq \mathrm{S} / \mathrm{D} \leq 3$ and a comparatively large increment is shown in range $3 \leq \mathrm{S} / \mathrm{D} \leq 4$. Value of increment of drag coefficient is further reduced in range of $4 \leq \mathrm{S} / \mathrm{D} \leq 5$.

Drag coefficient on cylinder 2 for a range $1 \leq \mathrm{S} / \mathrm{D} \leq 3$ shows negative value while for a range of $3 \leq \mathrm{S} / \mathrm{D} \leq 5$ it is converted from negative to positive. For a range of $1 \leq \mathrm{S} / \mathrm{D} \leq 2$ it shows a slight reduction in the value while it goes increases in the range of $2 \leq \mathrm{S} / \mathrm{D} \leq 3$.

For the range of $3 \leq \mathrm{S} / \mathrm{D} \leq 4$ it shows a sudden increment in the value of drag coefficient and also comes from negative to positive. For range of $4 \leq \mathrm{S} / \mathrm{D} \leq 5$ it goes for further increment.

It is interesting to note that lift coefficient for upstream cylinder has a very little change in the range of $1 \leq \mathrm{S} / \mathrm{D} \leq 3$ but has a large change in the range of $4 \leq \mathrm{S} / \mathrm{D} \leq 5$. This point is also remarkable that lift coefficient for upstream cylinder has a very low values comparative to downstream cylinder.

The variation of drag coefficient with respect to the flow time has been shown below for all values of $\mathrm{L} / \mathrm{D}$ and at $\mathrm{Re}=150$ as.

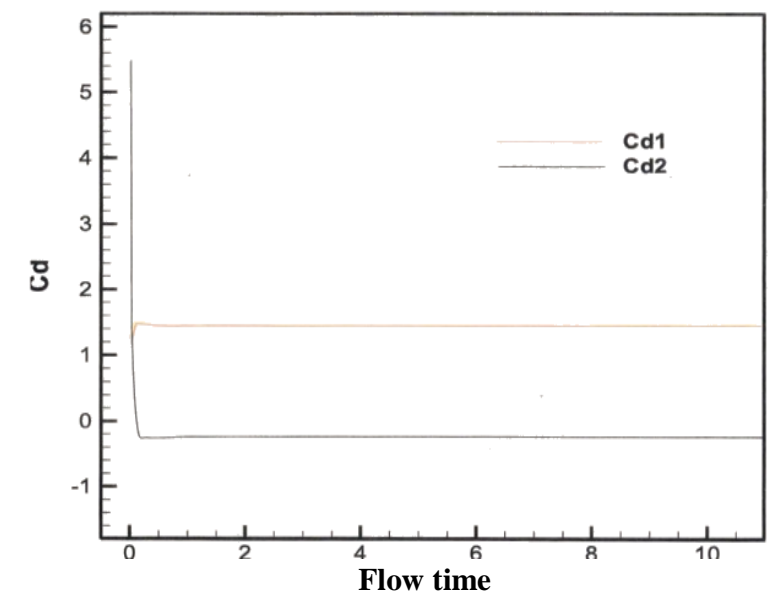

Fig 2. Variation of coefficient of drag for $S R=1$

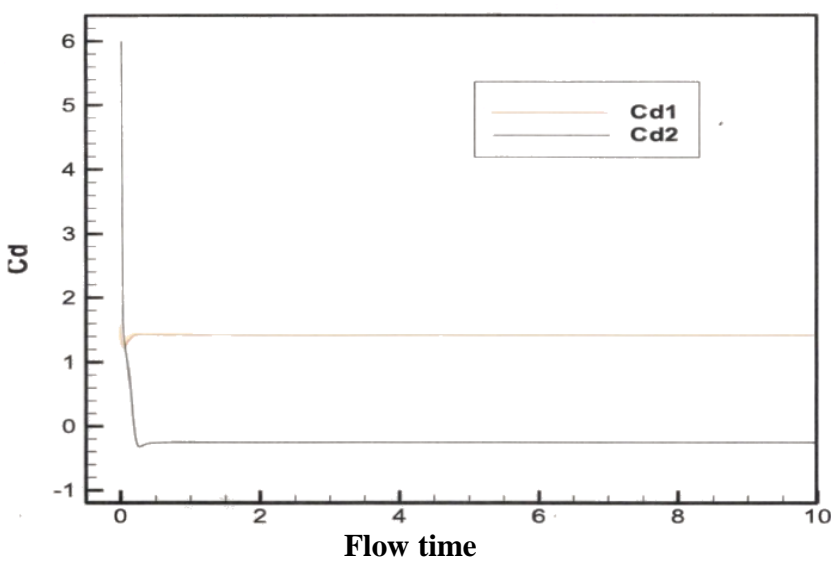

Fig 3. Variation of coefficient of drag for $S R=2$

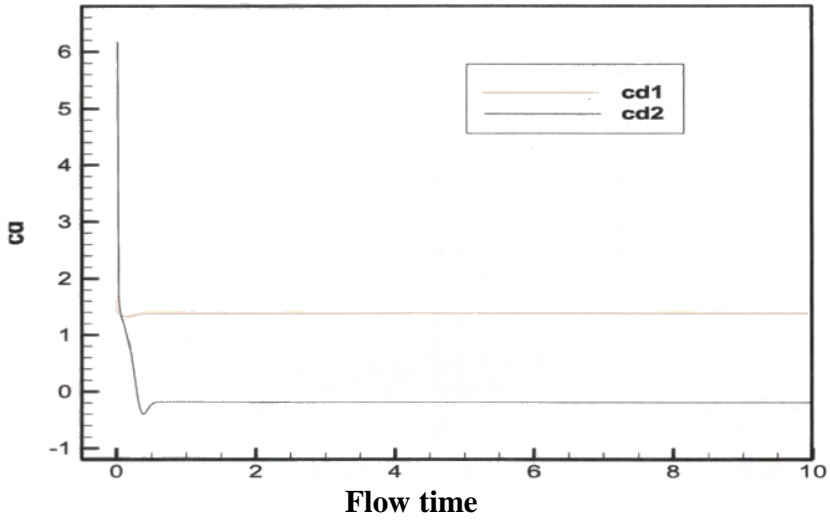

Fig 4. Variation of coefficient of drag for $S R=3$

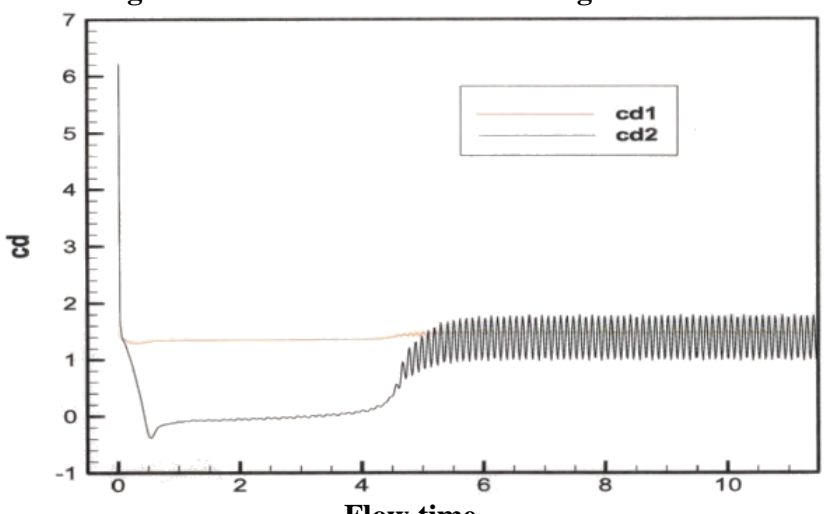

Flow time

Fig 5. Variation of coefficient of drag for $S R=4$

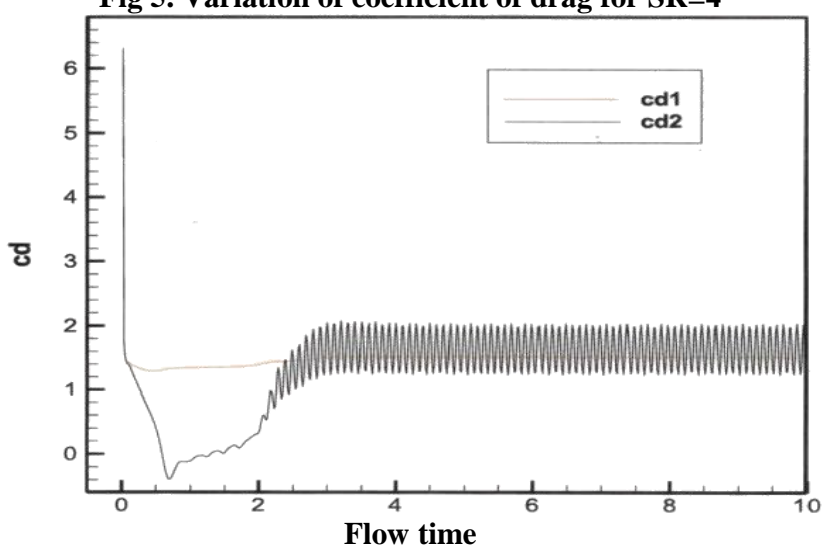

Fig 6. Variation of coefficient of drag for $S R=5$

The basis flow pattern at $\mathrm{Re}=150$ shows that vortex formation reduces and flow become more and more stable in 
ISSN: 2277-3754

ISO 9001:2008 Certified

International Journal of Engineering and Innovative Technology (IJEIT)

Volume 10, Issue 4, October 2020

the range of $1 \leq \mathrm{S} / \mathrm{D} \leq 3$ while it again become unstable in the range of $3 \leq \mathrm{S} / \mathrm{D} \leq 5$.

A. Variation of Lift Coefficient on Two Cylinders at Various Spacing Ratios

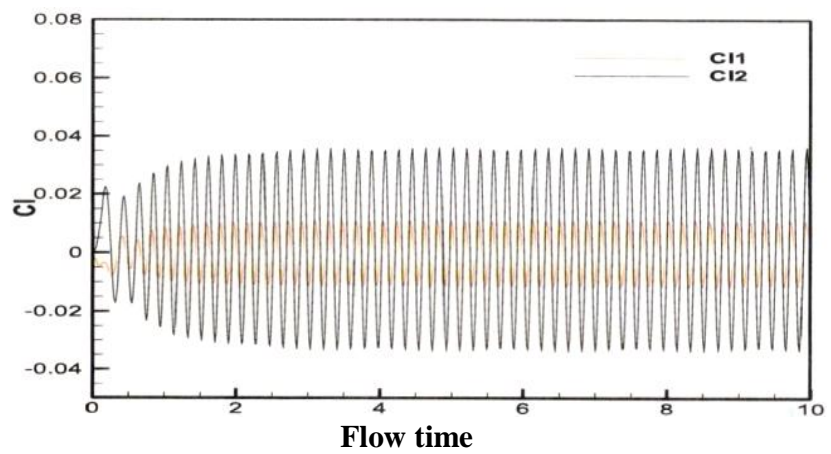

Fig 7. Variation of coefficient of lift for $S R=1$

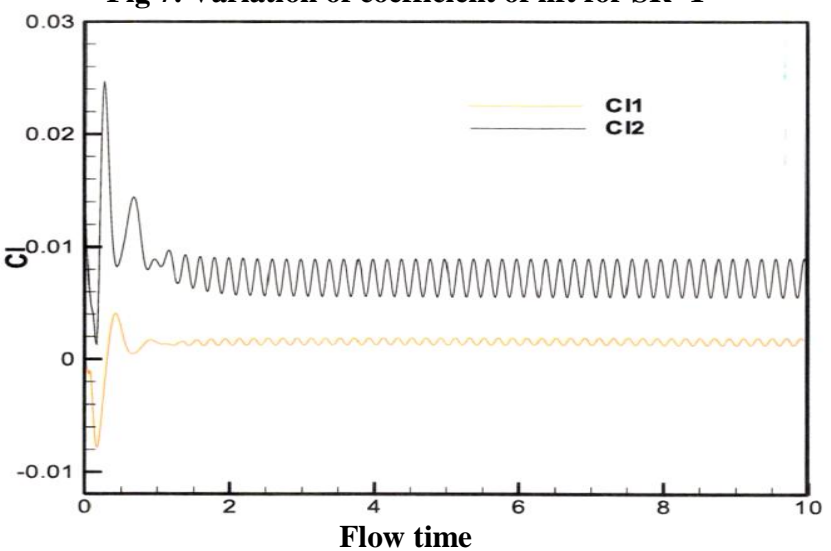

Fig 8. Variation of coefficient of lift for $S R=2$

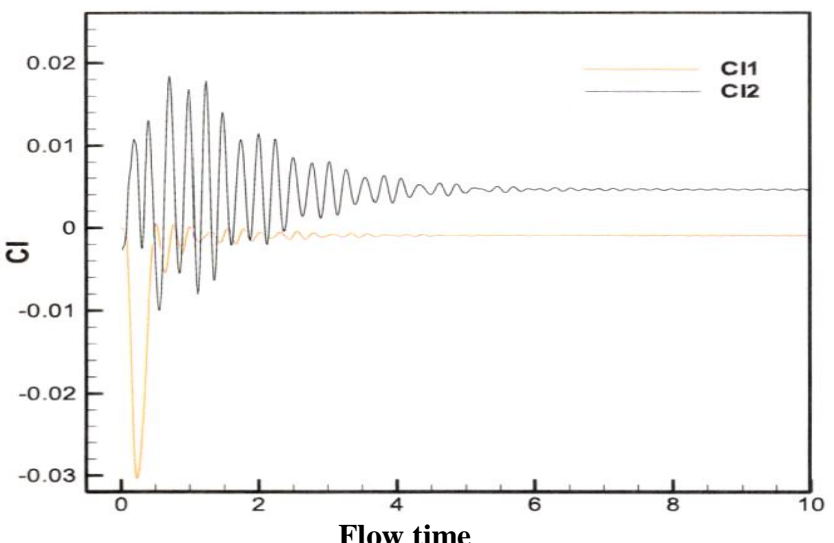

Fig .9. Variation of coefficient of lift for $S R=3$

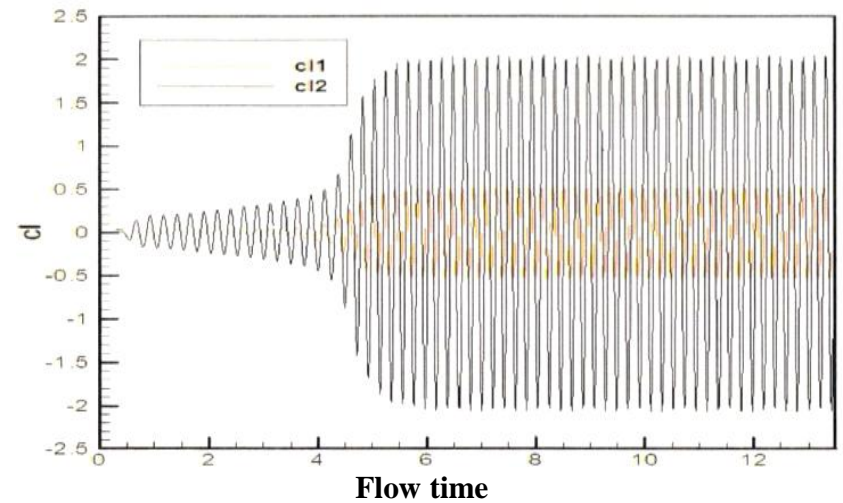

Fig 10. Variation of coefficient of lift for $S R=4$

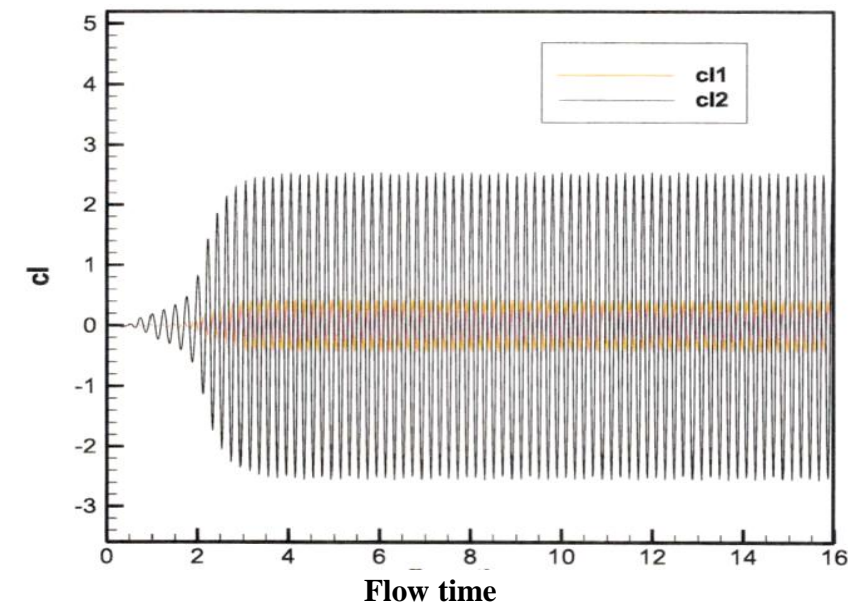

Fig 11. Variation of coefficient of lift for $S R=5$

The lift coefficient for the downstream cylinder increases for the range of $1 \leq \mathrm{S} / \mathrm{D} \leq 3$ as shown in above figures, but a very large change can be seen for spacing ratio of 4 and 5 . From figure 4.10 and figure 4.11 it can be noted that the variation in the lift coefficient is larger for the downstream cylinder as compared to the upstream cylinder in the spacing range of $4 \leq \mathrm{S} / \mathrm{D} \leq 5$. The scope of the lift coefficient for the downstream cylinder increases as the gap ratio between the two cylinders increases. The scope of the lift coefficient for the upstream cylinder is not larger as much as seen in the scope for the lift in the downstream cylinder.

\section{B. The details of the various velocity contours are shown below for different spacing ratio}

From below figure it can be concluded that an accelerated zone is formed between upstream cylinder and the downstream cylinder in the spacing range of the 1 to 3 . Contours in the below figure clearly shows that for spacing ratio 1 flow linearity lies in between 0 to 5 on scale while it goes liner upto 5 on scale for spacing ratio 2. For spacing ratio 3 flows goes linear upto 20 on scale. After this range the velocity contours starts to shed for both the cylinders and each cylinder behaves as the single cylinder in the wake of flowing fluid.
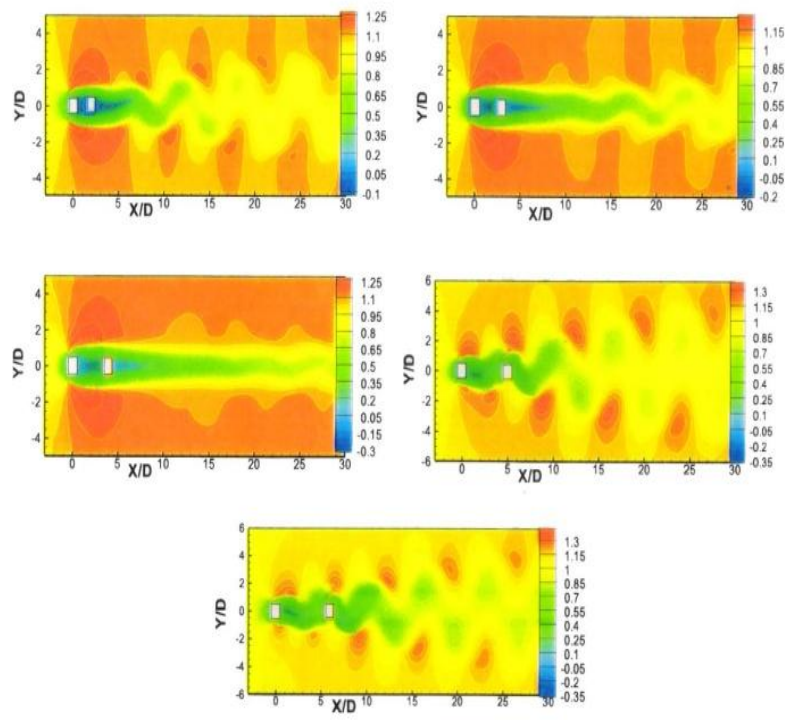

Fig 12 Various velocity contours for different spacing ratio 
ISSN: 2277-3754

\section{ISO 9001:2008 Certified \\ International Journal of Engineering and Innovative Technology (IJEIT) \\ Volume 10, Issue 4, October 2020}

C. The details of the various streamlines are shown below for different spacing ratio:
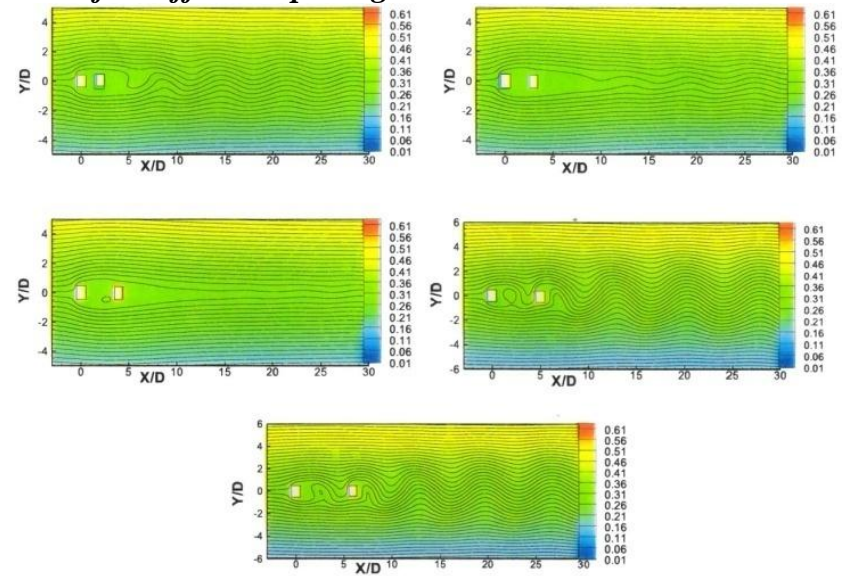

Fig 14 Various streamlines at different spacing ratio

The above fig shows the variations of streamlines at different spacing ratio. The streamlines at spacing ratio between $1 \leq \mathrm{L} / \mathrm{D} \leq 2$ passes away without creating any disturbance in between cylinders while at $S / D=3$ it shows a slight variation. It shows a huge disturbance in between the cylinders in the range $4 \leq \mathrm{S} / \mathrm{D} \leq 5$. This shows that the behaviour of upstream cylinder is similar to downstream cylinder in spacing range of 1 and 3 . In the spacing range of the 4 to 5 the circular zones are of the different types.

D. The details of the various Y-velocity contours are shown below for different spacing ratio
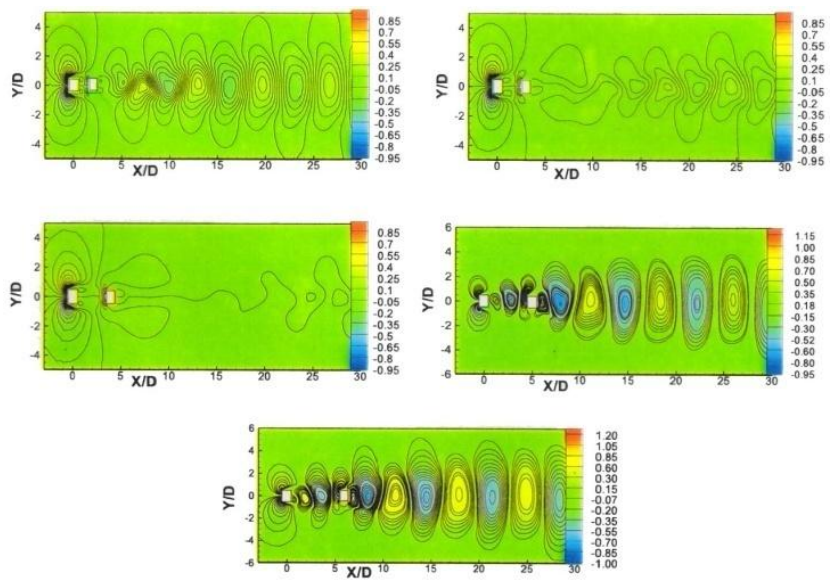

Fig 15 Various Y-velocity contours at different spacing ratio

The velocities normal to the velocities of direction of flow, i.e. along y-axis, are called as V-velocities. The v-velocity component is observed to be positive and negative alternatively. The solid lines represent the positive and the dashed lines represent the negative velocities. The above figure shows the $\mathrm{V}$-velocities for different spacing ratios (SR) ranging from 1 to 5 .

\section{CONCLUSION}

It can be concluded that vortex shedding for the spacing ratio of 4 is much similar to the shedding's occurred in the spacing ratio of 5 as shown below in Figure 4.12 to Figure 4.15. The nature of the shedding's in the spacing ratio of 1 and 2 have comparatively smaller vortex as compared to vortex formed in spacing ratio of 4 and 5 . hence it can be clearly concluded that the formation of the shadings are widely depends on the spacing ratio at fixed Reynolds number. Least shedding occurred at $\mathrm{S} / \mathrm{D}=3$.

Various values of strouhal number with graphical representation are also shown below.

Table 2. Spacing ratio (S/D) v/s Strouhal number

\begin{tabular}{|c|c|}
\hline S/D & Strouhal Number \\
\hline 1 & 0.130 \\
\hline 2 & 0.125 \\
\hline 3 & 0.115 \\
\hline 4 & 0.120 \\
\hline 5 & 0.125 \\
\hline
\end{tabular}

Spacing ratio (S/D) v/s Strouhal number

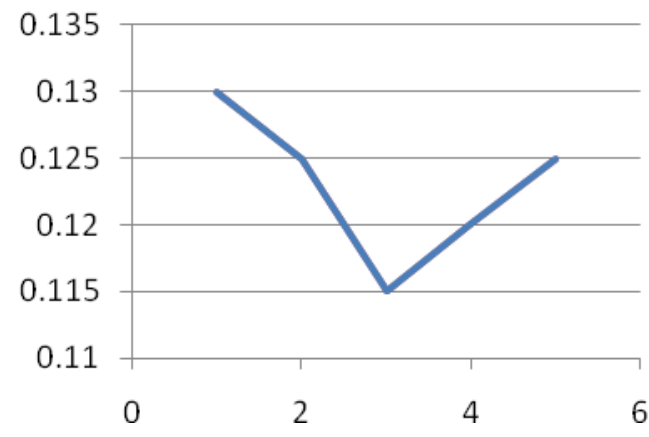

Fig 16.Spacing ratio (S/D) v/s Strouhal number

It is very important to note the change in Strouhal number that occurs due to the interference. A minimum value of $\mathrm{St}=$ 0.115 is reached for a gap of $S / D=3$. This value is of the Strouhal number is reduced by $12 \%$ as compared to spacing ratio of $\mathrm{S} / \mathrm{D}=1(\mathrm{St}=0.130)$. A minimum drag coefficient of upstream cylinder is founds at spacing ratio 3. Drag coefficient for the downstream cylinder at the same spacing ratio is found to be negative.

There is a lot of scope for future research in case of flow around square cylinder as it can be done on vibrating cylinders. Present research is done for two square cylinders in inline arrangement. This can be expanded to multiple cylinders to make this research more useful for better designing of electronic circuits and other similar objects. Present study is restricted to $2^{\text {nd }}$ analysis of flow over bluff bodies so in future research this can be expanded to $3 \mathrm{~d}$ analysis of flow over bluff bodies.

\section{ACKNOWLEDGMENT}

We would like to express our appreciation and gratitude to Mr. Devendra Sikarwar, Head of department, Mechanical Engineering, Patel college of Science and Technology Indore for his valuable support, advice, research facilities during this study work. Our special appreciation goes to Prof. Pradeep Singh Hada and Prof. Kapil Patodi of IPS Academy, Institute of Engineering and Science Indore (M.P.) for their continuous help and support.

Finally, I thank the Almighty God, without blessings of whose, nothing would be possible. 
ISSN: 2277-3754

\section{ISO 9001:2008 Certified}

International Journal of Engineering and Innovative Technology (IJEIT)

Volume 10, Issue 4, October 2020

\section{REFERENCES}

[1] M.P. Paidoussis, "A review of flow-induced vibrations in reactors and reactor components," Nuclear Engineering and Design vol. 74, Issue 1, pp. 31-60, January 1983.

[2] A. K. El Wahed, M. W. Johnson and J.L. Sproston, "Numerical study of vortex shedding from different shaped bluff bodies," Flow Meas. Instrument, vol. 4, Issue 4, pp:233-240,1993.

[3] C. Norberg, "Flow around rectangular cylinders: Pressure forces and wake frequencies", Journal of Wind Engineering and Industrial Aerodynamics, vol. 49, pp. 187-196, 1993.

[4] D. Yu, A. Kareem, "Two-dimensional simulation of flow around rectangular prisms," Journal of Wind Engineering and Industrial Aerodynamics vol. 62 pp. 131-161, 1996.

[5] A.K. Saha, G. Biswas, K. Muralidhar, "Numerical study of the turbulent unsteady wake behind a partially enclosed square cylinder using RANS," Computational Methods Application to Mechanical Engineering, pp. 323-341, 1999.

[6] Ian Taylor, Marco Vezza, "Prediction of unsteady flow around square and rectangular section cylinders using a discrete vortex method," Journal of Wind Engineering and Industrial Aerodynamics, vol. 82 pp. 247-269, 1999.

[7] A.K. Saha, K. Muralidhar, G. Biswas, "Vortex structures and kinetic energy budget in two- dimensional flow past a square cylinder," Computers \& Fluids vol. 29, pp.669-694, 2000.

[8] Chia-Hung Liu, Jerry M. Chen, "Observations of hysteresis in flow around two square cylinders in a tandem arrangement," Journal of Wind Engineering and Industrial Aerodynamics, vol. 90, pp: 1019-1050, 2002.

[9] R. Ajith Kumar, B.H.L. Gowda, "Flow-induced vibration of a square cylinder without and with interference," Journal of Fluids and Structures vol. 22 pp. 345-369, 2006.

[10] A. K. Dhiman , R. P. Chhabra , A. Sharma \& V. Eswaran, "Effects of Reynolds and Prandtl Numbers on Heat Transfer Across a Square Cylinder in the Steady Flow Regime," Numerical Heat Transfer, Part A: Applications: An International Journal of Computation and Methodology, 49:7, pp.717-731, 2006.

[11] Shashi Ranjan Kumar, Atul Sharma and Amit Agrawal, "Simulation of flow around a row of square cylinders," J. Fluid Mech. vol. 606, pp. 369-397, 2008.

\section{AUTHOR BIOGRAPHY}

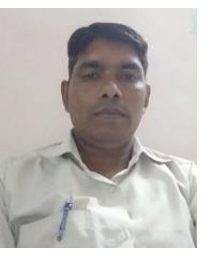

Ramchandar Verma is M.Tech. Student at Mechanical Engineering of Patel College of Science and Technology, Indore (affiliated to RGPV Bhopal). He received his BE Degree in 2015 in Mechanical Engineering from S.G.S.I.T.S. Indore. He is Currently Working on Effect of the Gap Ratio on the Flow Patterns of the Vortex Flow forming behind the two square cylinders in tandem arrangement.

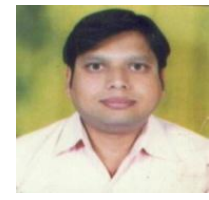

Dheeraj shriwas is an Assistant Professor in Mechanical Engineering of Patel College of Science and Technology, Indore (RGPV). He received his BE
Degree in 2008 in Mechanical Engineering from Patel College of Science and Technology Bhopal. He did his M.Tech. in Mechanical Engineering from B.M. college Indore. 